\title{
Control of Encapsulation Efficiency and Initial Burst in Polymeric Microparticle Systems
}

\author{
Yoon Yeo and Kinam Park \\ Purdue University, Departments of Pharmaceutics and Biomedical Engineering, West Lafayette, IN 47907, USA
}

(Received November 20, 2003)

\begin{abstract}
Initial burst is one of the major challenges in protein-encapsulated microparticle systems. Since protein release during the initial stage depends mostly on the diffusional escape of the protein, major approaches to prevent the initial burst have focused on efficient encapsulation of the protein within the microparticles. For this reason, control of encapsulation efficiency and the extent of initial burst are based on common formulation parameters. The present article provides a literature review of the formulation parameters that are known to influence the two properties in the emulsion-solvent evaporation/extraction method. Physical and chemical properties of encapsulating polymers, solvent systems, polymer-drug interactions, and properties of the continuous phase are some of the influential variables. Most parameters affect encapsulation efficiency and initial burst by modifying solidification rate of the dispersed phase. In order to prevent many unfavorable events such as pore formation, drug loss, and drug migration that occur while the dispersed phase is in the semi-solid state, it is important to understand and optimize these variables.
\end{abstract}

Key words: PLGA, Microparticle, Encapsulation efficiency, Release profile, Initial burst, Formulation parameters

\section{INTRODUCTION}

Major challenges in developing protein-encapsulated microparticle systems are (i) instability of encapsulated proteins, (ii) their incomplete release, and (ii) initial burst release. It is widely known that the chemical and mechanical stresses produced during the microencapsulation process and the release period exert damaging effects on the conformational and biological integrity of the protein (Park et al., 1998; van de Weert et al., 2000a; Schwendeman, 2002). In addition, the proteins, which are exposed to the acidic and/or hydrophobic microenvironments within the degrading polymer for a long period of time, undergo irreversible aggregation and/or degradation and non-specific adsorption and become unavailable for release (Schwendeman, 2002; Zhu and Schwendeman, 2000; Lu and Park, 1995). In this way, the protein instability issue is closely related to the incomplete release issue. It

Correspondence to: Kinam Park, Ph.D., Purdue University, School of Pharmacy, 575 Stadium Mall Drive, Room G22, West Lafayette, IN 47907-2051, USA

Tel: 1-765-494-7759, Fax: 1-765-496-1903

E-mail: kpark@purdue.edu is also frequently seen that many controlled release formulations including protein-loaded microparticles release a large bolus of drugs before they can reach a stable release rate (i.e., burst release or initial burst) (Huang and Brazel, 2001). In most cases, the burst release is an ineffective form of drug usage from both therapeutic and economic standpoints. For this reason, one of the goals in development of protein microencapsulation systems is to reduce the initial burst and achieve a constant release rate thereafter.

The present article focuses on reviewing previous research conducted to control the release profile from polymeric systems such as poly (lactic-co-glycolic acid) (PLGA) microparticles. Most examples discussed here are from the emulsion-solvent evaporation/extraction method, unless specified otherwise.

Protein release from the PLGA microparticles during the initial release stage depends on diffusional escape of the protein through channels existing in the polymer matrix. Given that the time required until the onset of PLGA degradation ranges from weeks to months (Table I-III), drug release during the first few days depends on how successfully the diffusion is controlled. In most cases, the 
Table I. Degradation properties of microparticles prepared from PLGA (50/50) (Mehta et al., 1994)

\begin{tabular}{llc}
\hline Mw & Bulk density & $\mathrm{T}_{\text {onset }}$ (weeks) \\
\hline 29,049 & 0.094 (porous) & 1.5 \\
25,643 & 0.167 (less porous, but radiated) & 3.0 \\
33,092 & 0.167 (less porous, intact) & 4.3 \\
\hline
\end{tabular}

-No purchase information

Table II. Time taken for a $50 \%$ reduction in the weight of PLGA microparticles $\left(t_{50 \%}\right)$ (O'Hagan et al., 1994)

\begin{tabular}{lcc}
\hline LA/GA & Molecular weight & $t_{50 \%}$ (days) \\
\hline $50 / 50$ (Resomer $^{\circledR}$ RG503) & $33 \mathrm{kDa}$ & 25 \\
$50 / 50$ (Resomer $^{\circledR}$ RG506) & $84 \mathrm{kDa}$ & 50 \\
$75 / 25$ (Resomer $^{\circledR}$ RG755) & $83 \mathrm{kDa}$ & 270 \\
\hline
\end{tabular}

-Resomer ${ }^{\circledR}$ : Boehringer Ingelheim

Table III. Polymers from Alkermes ${ }^{\circledR}$

\begin{tabular}{lccc}
\hline Medisorb ${ }^{\circledR}$ polymers & $\begin{array}{c}\text { Inherent viscosity } \\
(\mathrm{dL} / \mathrm{g})\end{array}$ & LA/GA & $\begin{array}{c}\text { Degradation } \\
\text { information }\end{array}$ \\
\hline 100 DL HIGH IV & $0.66-0.80$ & $100 / 0$ & $12-16$ months \\
100 DL LOW IV & $0.50-0.65$ & & \\
\hline 8515 DL HIGH IV & $0.66-0.80$ & $85 / 15$ & $5-6$ months \\
8515 DL LOW IV & $0.50-0.65$ & & \\
\hline 7525 DL HIGH IV & $0.66-0.80$ & $75 / 25$ & $4-5$ months \\
7525 DL LOW IV & $0.50-0.65$ & & \\
\hline 6535 DL HIGH IV & $0.66-0.80$ & $65 / 35$ & $3-4$ months \\
6535 DL LOW IV & $0.50-0.65$ & & \\
\hline 5050 DL HIGH IV & $0.66-0.80$ & $50 / 50$ & $1-2$ months \\
5050 DL LOW IV & $0.50-0.65$ & & \\
\hline 5050 DL 1A & $0.08-0.12$ & $50 / 50$ & $1-2$ weeks \\
5050 DL 2A & $0.13-0.20$ & & $2-3$ weeks \\
5050 DL 2.5A & $0.21-0.31$ & & $2-4$ weeks \\
5050 DL 3A & $0.25-0.43$ & & $3-4$ weeks \\
5050 DL 4A & $0.38--0.48$ & & $3-4$ weeks \\
\hline
\end{tabular}

-50/50 polymers with "A" (i.e., $5050 \mathrm{DL} 1 \mathrm{~A}$ ) have a carboxyl end group; the rest polymers have an alkyl ester end group (Alkermes).

burst release is due to poor control over the diffusionbased release in this stage. The degree of initial burst from the microparticles depends on the ability of the polymer matrix to encapsulate the protein, thereby making it unavailable for immediate diffusion (Mehta et al., 1996). For this reason, efforts to reduce the initial burst have followed in the same track as those to increase encapsulation efficiency. Although the increase in encapsulation efficiency does not necessarily lead to reduction of the burst release, understanding the previous effort to maximize the encapsulation efficiency will thus be useful in controlling the release profile.

\section{MATERIALS AND METHODS}

\section{Formulation parameters relevant to encapsula- tion efficiency}

Most attempts to increase encapsulation efficiency are based on a common idea that fast polymer precipitation on the surface of the dispersed phase can prevent drug loss into the continuous phase (Bodmeier and McGinity, 1988). On the other hand, when solidification of the dispersed phase is delayed, encapsulation efficiency becomes low because more drugs diffuse into the continuous phase (Fig. 1). Fig. 2 summarizes the formulation parameters that influence polymer precipitation and encapsulation efficiency.

\section{Solubility of polymer in organic solvent}

According to Mehta et al., solubilities of polymers in organic solvents determines the solidification rate of the polymers during the microparticle preparation process, which in turn affects microparticle properties such as drug incorporation, matrix porosity, and solvent residues (Mehta et al., 1996). Eleven low molecular weight PLGA (ratio of

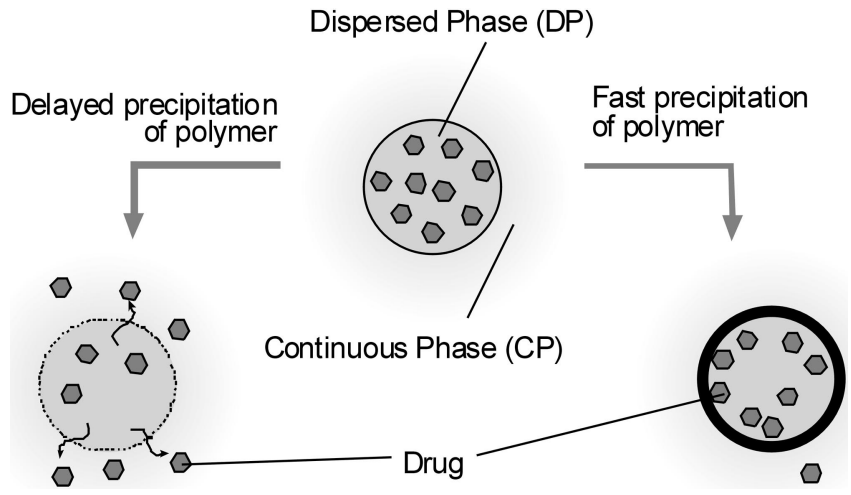

Fig. 1. Schematic description of the rationale for encapsulation efficiency.

\begin{tabular}{|l|l|}
\hline $\begin{array}{l}\text { High solubility of polymer in organic } \\
\text { solvent } \\
\text { water }\end{array}$ & $\begin{array}{l}\text { Low solubility of polymer in organic } \\
\text { solvent }\end{array}$ \\
Low concentration of polymer & $\begin{array}{l}\text { High solubility of organic solvent in } \\
\text { water }\end{array}$ \\
High DP/CP ratio & High concentration of polymer \\
Slow solvent removal rate & Low DP/CP ratio \\
\hline \begin{tabular}{|l|} 
Slow solidification \\
of microparticles
\end{tabular} \\
\hline
\end{tabular}

Fig. 2. Factors influencing encapsulation efficiency 
lactide to glycolide residue (L/G ratio) $=50 / 50$ ) and PLGA $(\mathrm{L} / \mathrm{G}$ ratio $=75 / 25)$ polymers from three manufacturers were chosen for making microparticles by the emulsionsolvent extraction method, and the relationship between properties of polymer and that of the resulting microparticles were investigated. Properties of polymers that were compared are $\mathrm{L} / \mathrm{G}$ ratio, molecular weight, and their end structures.

Here, solubilities of the polymers in methylene chloride were compared by measuring the methanol cloud point $\left(C_{s}\right)$ : Higher $C_{s}$ meant that the polymer was more soluble in methylene chloride and, thus, required a greater amount of methanol to precipitate from the polymer solution. The PLGA polymer of a relatively high L/G ratio (75/25) had a higher solubility in methylene chloride than the other PLGA (L/G ratio=50/50). A lower molecular weight polymer had a higher solubility in methylene chloride than a higher molecular weight polymer. End-capped polymers, which were more hydrophobic than non-end-capped polymers of the same molecular weight and component ratio, were more soluble in methylene chloride.

Diffusion of drugs into the continuous phase mostly occurred during the first 10 minutes of emulsification; therefore, as the time the polymer phase stayed in the non-solidified (semi-solid) state was extended, encapsulation efficiency became relatively low. In Mehta's study, polymers having relatively high solubilities in methylene chloride took longer to solidify and resulted in low encapsulation efficiencies, and vice versa (Mehta et al., 1996). Particle size and bulk density also varied according to the polymer. Since polymers having higher solubilities in methylene chloride stayed longer in the semi-solid state, the dispersed phase became more concentrated before it completely solidified, resulting in denser microparticles.

In different literature, it was shown that the use of relatively hydrophilic PLGA which carried free carboxylic end groups (Resomer ${ }^{\circledR} R G 502 H, L / G=50 / 50$ ) resulted in a significantly higher encapsulation efficiency compared to that of an end-capped polymer (Resormer ${ }^{\circledR}$ RG502, L/G= 50/50) (Johansen et al., 1998). A similar explanation as above applies to this observation: Hydrophilic PLGA is relatively less soluble in the solvent, methylene chloride, and precipitates more quickly than the end-capped one. High solidification rate might have increased the encapsulation efficiency. On the other hand, the authors attribute the increase to the enhanced interaction between PLGA and the protein through hydrogen bonding and polar interactions (Johansen et al., 1998). Walter et al. also observe an increase in encapsulation efficiency from using relatively hydrophilic PLGA (Resomer ${ }^{\circledR}$ RG502H) in DNA microencapsulation (Walter et al., 2001). Here, the authors suggest that the hydrophilicity of the polymer enhanced the stability of the primary emulsion, and it contributed to such an increase.

Similarly, among the polyvinyl alcohol (PVA)-graft-PLGA polymers having different sizes of PLGA segments, the polymers containing shorter PLGA segments, which were hence more hydrophilic, were found to be more efficient in encapsulating hydrophilic macromolecules (Frauke Pistel et al., 2001). Decrease in the length of PLGA segments resulted in increase of encapsulation efficiency from 82 to $97 \%$ for FITC-dextran and from 66 to $80 \%$ for BSA. The contribution of the hydrophilicity can be, again, interpreted in two ways: First, the increasing hydrophilicity reduced solubility of the polymer in methylene chloride, and caused fast polymer solidification. Alternatively, the relatively hydrophilic polymer allowed efficient internalization of the hydrophilic macromolecules within the microparticles (Frauke Pistel et al., 2001).

\section{Solubility of organic solvent in water}

On the other hand, Bodmeier et al. found that methylene chloride resulted in a higher encapsulation efficiency as compared with chloroform or benzene, even though methylene chloride was a better solvent for poly (lactic acid) (PLA) than the others (Bodmeier and McGinity, 1988). In order to justify this discrepancy, the authors point out that methylene chloride is more soluble in water than chloroform or benzene. The 'high' solubility allowed relatively fast mass-transfer between the dispersed and the continuous phases and led to fast precipitation of the polymer. The significance of solubility of the organic solvent in water was also confirmed by the fact that the addition of water-miscible co-solvents such as acetone, methanol, ethyl acetate, or dimethyl sulfoxide (DMSO), contributed to increase of the encapsulation efficiency (Bodmeier and McGinity, 1988). Knowing that the methanol is a non-solvent for PLA and a water-miscible solvent, it can be assumed that methanol played a dual function in facilitating the polymer precipitation: First, the presence of methanol in the dispersed phase decreased the polymer solubility in the dispersed phase (Jeyanthi et al., 1997). Second, as a water-miscible solvent, methanol facilitated diffusion of water into the dispersed phase.

In order to explain the low encapsulation efficiency obtained with benzene, the authors mention that the benzene required a larger amount of water (non-solvent) than methylene chloride for precipitation of the polymer, and the drug was lost due to the delayed solidification. However, given that benzene is a poorer solvent than methylene chloride for a PLA polymer, this argument does not agree with the widely spread idea that a poor solvent requires a smaller amount of non-solvent to precipitate a polymer. In fact, there could have been a better explanation if they had considered that the delayed solidification was due to the low solubility of benzene in water: As a poor 
solvent for a PLA polymer, benzene requires only a small amount of non-solvent for complete solidification of the polymer. However, since benzene can dissolve only a tiny fraction of water, it takes much longer to uptake water into the dispersed phase. That is, while solubility of a polymer in an organic solvent governs the quantity of a nonsolvent required in precipitating a polymer, solubility of the organic solvent in the non-solvent limits diffusion of the non-solvent into the polymer phase. Thus, when a cosolvent system is involved, both solubility of a polymer in a solvent and solubility of the solvent in a non-solvent participate in determining the solidification rate of the dispersed phase.

The importance of the solvent system was demonstrated in another example (Park et al., 1998). Lysozyme-loaded PLGA microparticles were prepared using the oil in water $(\mathrm{o} / \mathrm{w})$ single emulsion technique. Here, the authors used a co-solvent system, varying the ratio of the component solvents. DMSO was used for solubilization of lysozyme and PLGA, and methylene chloride was used for generation of emulsion drops as well as solubilization of PLGA. Encapsulation efficiency increased, and initial burst decreased as the volume fraction of DMSO in the co-solvent system increased. Particle size increased, and density of the microparticle matrix decreased with increasing DMSO. Overall, these results indicate that the presence of DMSO increased the hydrophilicity of the solvent system and allowed fast extraction of the solvent into the continuous phase, which led to higher encapsulation efficiency and larger particle size.

\section{Polymer concentration}

Encapsulation efficiency increases with increasing polymer concentration (Mehta et al., 1996; Rafati et al., 1997; Li et al., 1999). For example, the encapsulation efficiency increased from 53.1 to $70.9 \%$ when concentration of the polymer increased from 20.0 to $32.5 \%$ (Mehta et al., 1996). High viscosity and fast solidification of the dispersed phase contributed to reducing porosity of the microparticles as well (Schlicher et al., 1997).

The contribution of a high polymer concentration to the encapsulation efficiency can be interpreted in two ways. First, when highly concentrated, the polymer precipitates faster on the surface of the dispersed phase and prevents drug diffusion across the phase boundary (Rafati et al., 1997). Second, the high concentration increases viscosity of the solution and delays the drug diffusion within the polymer droplets (Bodmeier and McGinity, 1988).

\section{Ratio of dispersed phase to continuous phase (DP/ CP ratio)}

Encapsulation efficiency and particle size increase as the volume of the continuous phase increases ( $\mathrm{Li}$ et al.,
1999, Mehta et al., 1996). For example, the encapsulation efficiency increased more than twice as the ratio of the dispersed phase to the continuous phase (DP/CP ratio) decreased from $1 / 50$ to $1 / 300$ (Mehta et al., 1996). It is likely that a large volume of continuous phase provides a high concentration gradient of the organic solvent across the phase boundary by diluting the solvent, leading to fast solidification of the microparticles. A relevant observation is described in the literature (Sah, 1997). In this example, which utilized ethyl acetate as a solvent, the formation of microparticles was dependent on the volume of the continuous phase. When $8 \mathrm{~mL}$ of PLGA solution (o) was poured into 20 or $50 \mathrm{~mL}$ of water phase $(w)$, the polymer solution was well disintegrated into dispersed droplets. On the other hand, when the continuous phase was $80 \mathrm{~mL}$ or more, the microspheres hardened quickly and formed irregular precipitates. This is because the large volume of continuous phase provided nearly a sink condition for ethyl acetate and extracted the solvent instantly.

Due to the fast solidification of the polymer, particle size increased with increasing volume of the continuous phase. Microparticles generated from a low DP/CP ratio had a lower bulk density $(0.561 \mathrm{~g} / \mathrm{cc}$ at $1 / 50 \mathrm{vs.} 0.357 \mathrm{~g} / \mathrm{cc}$ at $1 /$ 300 ), which the authors interpret as an indication of higher porosity of the polymer matrix (Mehta et al., 1996). On the other hand, a different example shows that a higher DP/ $\mathrm{CP}$ ratio resulted in increased porosity, providing a large specific surface area (measured by the BET method) and the scanning electron microscope (SEM) pictures as evidence (Jeyanthi et al., 1997). This apparent discrepancy can be explained by the fact that low bulk density (Mehta et al., 1996) is not a true reflection of porosity but a result of large particle size. In fact, porosity increases with increasing DP/CP ratio, i.e., decreasing rate of the polymer precipitation. This will be discussed later.

\section{Rate of solvent removal}

The method and rate of solvent removal influence the solidification rate of the dispersed phase as well as morphology of the resulting microparticles (Mehta et al., 1994). In the emulsion-solvent evaporation/extraction method, the solvent can be removed by (i) evaporation, in which the solvent is evaporated around its boiling point or (ii) extraction into the continuous phase. The rate of solvent removal can be controlled by the temperature ramp or the evaporation temperature in the former and by the volume of the dilution medium in the latter.

PLGA microparticles containing salmon calcitonin (SCT) were prepared by emulsification, followed by different solvent removal processes (Mehta et al., 1994, Jeyanthi et al., 1996). In the temperature dependent solvent removal process, the solvent (methylene chloride) was removed by increasing the temperature from 15 to $40^{\circ} \mathrm{C}$ at different 
rates. The microparticles that resulted from this process had a hollow core and a porous wall. The core size and wall thickness were dependent on the temperature ramp: A rapid rise in temperature resulted in a thin wall and a large hollow core, whereas a stepwise temperature rise ( 15 to 25 , then to $40^{\circ} \mathrm{C}$ ) resulted in a reduced core size. It is believed that the hollow core was due to the rapid expansion of methylene chloride entrapped within the solidified microparticles. In controlled extraction of the solvent, the solvent was removed gradually and slowly by dilution of the continuous phase, which left the microparticles in the soft state for a longer period of time. The resulting microparticles showed a highly porous honeycomb-like internal structure without a hollow core. In the later study, it was noted that the porosity was a function of the amount of water diffused into the dispersed phase from the continuous phase, which could only be allowed before the dispersed phase solidified completely ( $\mathrm{Li}$ et al., 1995). In other words, the high porosity of the microparticles was due to the slow solidification of the microparticles.

Even though it is generally assumed that fast polymer solidification results in high encapsulation efficiency, this does not apply to the observation of Yang et al. (Yang et al., 2000). Here, the encapsulation efficiency was not affected by the solvent evaporation temperature. It is supposed that the different processing temperatures influenced not only the rate of polymer solidification but also the diffusivity of the protein and its solubility in water. While the high temperature facilitated solidification of the dispersed phase, it enhanced diffusion of the protein into the continuous phase, compromising the positive effect from the fast solidification.

\section{Interaction between drug and polymer}

Interaction between protein and polymer contributes to increasing encapsulation efficiency (Boury et al., 1997). Generally, proteins are capable of ionic interactions and are better encapsulated within polymers that carry free carboxylic end groups than the end-capped polymers. On the other hand, if hydrophobic interaction is a dominant force between the protein and the polymer, relatively hydrophobic end-capped polymers are more advantageous in increasing encapsulation efficiency (Mehta et al., 1996). For example, encapsulation efficiencies of more than $60 \%$ were achieved for salmon calcitonin (sCT) microparticles despite the high solubility of SCT in the continuous phase (Jeyanthi et al., 1997). This is attributed to the strong affinity of $\mathrm{SCT}$ to hydrophobic polymers such as PLGA. On the other hand, such interactions between protein and polymer can limit protein release from the microparticles (Park et al., 1998; Crotts and Park, 1997; Kim and Park, 1999).

In certain cases, a co-encapsulated excipient can mediate the interaction between protein and polymer (Johansen et al., 1998). Encapsulation efficiency increased when gammahydroxypropylcyclodextrin ( $\gamma$-HPCD) were co-encapsulated with tetanus toxoid in PLGA microparticles. It is supposed that the $\gamma$-HPCD increased the interaction by accommodating amino acid side groups of the toxoid into its cavity and simultaneously interacting with PLGA through van der Waals and hydrogen bonding forces.

\section{Solubility of drug in continuous phase}

Drug loss into the continuous phase occurs while the dispersed phase stays in a transitional, semi-solid state. If the solubility of the drug in the continuous phase is higher than in the dispersed phase, the drug will easily diffuse into the continuous phase during this stage. For example, the encapsulation efficiency of quinidine sulfate was 40 times higher in the alkaline continuous phase $(\mathrm{pH} 12$, in which quinidine sulfate is insoluble) than in the neutral continuous phase $(\mathrm{pH} 7$, in which quinidine sulfate is very soluble) (Bodmeier and McGinity, 1988). In another example, the encapsulation efficiency of rhodium(II) $(\mathrm{Rh}(\mathrm{II}))$ citrate in PLGA or poly(anhydride) was significantly enhanced after complexation with hydroxypropyl- $\beta$-cyclodextrin (HPBCD) (Sinisterra et al., 1999). It is likely that the complexation of the Rh(II) citrate with the HPBCD complex decreased the hydrophilicity of the drug and, thus, improved the retention of the drug in the dispersed phase. Indeed, the encapsulation efficiency increased from 30\% to $83 \%$ in PLGA microparticles and $22 \%$ to $79 \%$ in poly(anhydride) microparticles when free $\mathrm{Rh}(\mathrm{II})$ citrate was replaced with $\mathrm{Rh}$ (II) citrate-HPBCD complexes.

Protein is a typical example of a water soluble drug, of which efficient encapsulation remains a challenge. For optimization of encapsulation efficiency, making a waterinsoluble protein-zinc complex may be a reasonable approach (Tracy, 1998).

\section{Stability of primary emulsion}

In the double emulsion-solvent evaporation/extraction method, stability of the primary (water $\left(w_{1}\right)$ in oil (o), $w_{1} / 0$ ) emulsion is a critical factor for efficient internalization of the active ingredient (Nihant et al., 1994; Nihant et al., 1995; Schugens et al., 1994; Maa and Hsu, 1997). When the primary emulsion is unstable, encapsulation efficiency is low because the internal aqueous phase $\left(w_{1}\right)$ tends to merge with the neighboring aqueous continuous phase $\left(w_{2}\right)$. Stability of the primary emulsion can be enhanced by including emulsifying agents such as BSA (Schugens et al., 1994), PVA (Yang et al., 2001), Tween-80, or Span-80 (Li et al., 1999), either in the internal aqueous phase $\left(w_{1}\right)$ or in the polymer phase (o).

For example, encapsulation efficiency of indigocarmine (a dye marker) increased with the increasing use of BSA 
in the internal aqueous phase $\left(w_{1}\right)$, which stabilized the primary emulsion efficiently (Schugens et al., 1994). In another example, a high concentration of PVA included in the internal water phase led to increase in encapsulation efficiency and decrease in initial burst (Yang et al., 2001). Here, the encapsulated drug, BSA, was found to be uniformly distributed within the microparticles. This indicates that the PVA contributed to stabilization of the primary emulsion and efficient internalization of the protein. However, encapsulation efficiency decreased with increasing BSA loadings. Apparently, this result contrasts with the previous example because if the explanation in the former case were to apply to the latter, the increasing BSA content should stabilize the primary emulsion and increase the encapsulation efficiency. In fact, this discrepancy is due to the large concentration gradient of BSA. In the latter example, the increasing amount of BSA might have indeed increased the emulsion stability, but it also increased the concentration gradient of BSA between the dispersed phase and the continuous phase and provided a driving force for the diffusion of BSA into the continuous phase during polymer precipitation.

Volume and viscosity of the internal aqueous phase are often influential factors in determining encapsulation efficiency. Small volume (Li et al., 1999; Schlicher et al., 1997) and high viscosity (Ogawa et al., 1988; Maa and Hsu, 1997) are preferable in achieving high encapsulation efficiency. It is likely that the two factors also contribute to creating stable primary emulsion.

\section{Causes of initial burst release}

Initial burst release of protein from microparticles is generally explained in two ways. First, burst release occurs mainly due to the heterogeneous drug distribution. Proteins that are either loosely associated with the surface or embedded in the surface layer are responsible for the burst release (Sah et al., 1994; O'Hagan et al., 1994; Igartua et al., 1997; Rafati et al., 1997). Second, morphology of the microparticles causes initial burst: The drugs escape from the polymeric matrices through the pores and cracks that form during the microparticle fabrication process (Huang and Brazel, 2001; Yang et al., 2001).

Surface-associated drugs are widely known as main causes for the initial burst, but direct evidence is seldom found in the literature. On the contrary, a confocal microscopic analysis indicates that lysozyme encapsulated within PLGA microparticles by the double emulsionsolvent evaporation method existed exclusively inside the microparticles and distributed in several cavities within the microparticles (van de Weert et al., 2000b). Infrared imaging, Attenuated Total Reflectance-Fourier transform infrared spectrometry (FTIR), and FIIR-photoacoustic detection also supported the fact that the protein was

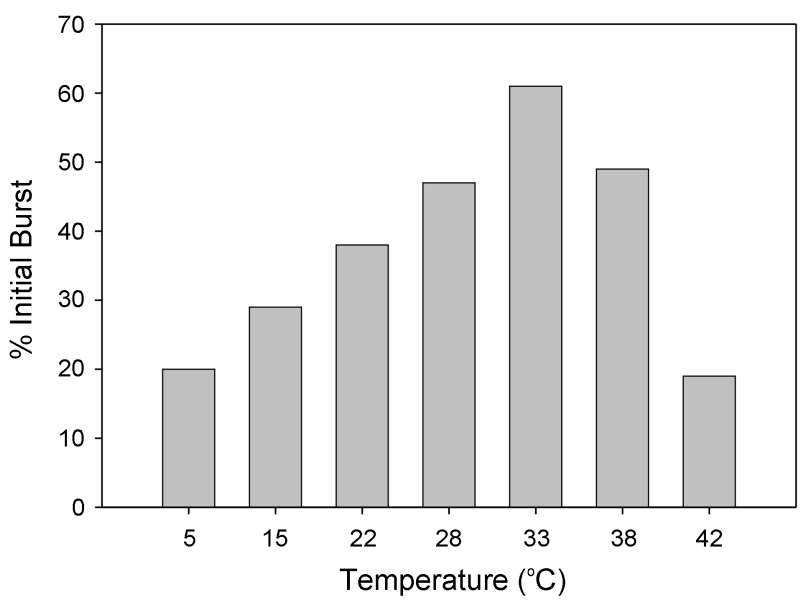

Fig. 3. Initial burst release as a function of preparation temperature. Data from Reference (Yang et al., 2000). Microparticles were prepared at different temperatures using the double emulsion-solvent evaporation/ extraction method.

distributed throughout the PLGA matrix, not concentrated on the surface. The authors concluded that the initial burst ( $50 \%)$ may have been caused by the diffusion of the protein through water-filled pores in the PLGA matrix rather than by the surface-bound protein.

It is likely that both causes contribute to the initial burst, although their relative contributions are yet to be determined. For example, Yang et al. used both explanations to interpret their observations (Yang et al., 2000). Microparticles were prepared at different temperatures using the double emulsion-solvent evaporation/extraction method. According to the previous discussion, the microparticles produced at lower temperatures would be more porous due to delayed solidification and diffusion of the aqueous phase into the dispersed phase. Therefore, it is expected that a lower fabrication temperature would result in a higher initial burst. However, both the lowest and highest temperatures yielded low initial bursts, and the highest initial burst occurred at an intermediate temperature (Fig. 3). Here, the authors point out that the temperature affected both drug distribution and morphology. At high temperatures, BSA diffused toward the surface of the microparticles, but fast solidification made the polymer relatively dense and reduced release of the surface-bound protein. At low temperatures, initial burst occurred due to the increased porosity, but only to a modest degree, since the low temperature prevented protein from moving toward the surface during solidification, leaving less protein near the surface. At $33^{\circ} \mathrm{C}$, the temperature was high enough to facilitate migration of the protein toward the surface and low enough to make the porous structure.

In the present article, discussion is made on the initial burst focusing more on morphology aspect simply because it is more relevant to the foregoing discussion and also 
relatively well-controlled.

\section{Porosity vs. density}

In many cases, the fact that microparticles are highly porous does not necessarily mean that the particle matrices are loose. It can be confusing since one may easily assume that high porosity is equivalent to low density. In order to straighten out this confusion, it is necessary to clarify the usage of two definitions in describing microparticle morphology: "porosity" and "density".

High porosity is generally a result of slow solidification. When the period that the microparticles remain soft is extended, the microparticles are allowed to shrink further and form smaller and denser matrices. At the same time, water from the internal aqueous phase $\left(\mathrm{w}_{1}\right)$ and/or the continuous phase $\left(\mathrm{w}_{2}\right)$ can diffuse into the dispersed phase to create water pockets and leave channels and pores within the polymer matrix ( $\mathrm{Li}$ et al., 1995; Yang et al., 2000). The end product of delayed solidification is a particle consisting of a dense polymeric matrix with pores or channels created by infused water (Fig. 4-A). In this case, the size of pores should be the same magnitude as the microparticle itself.

On the other hand, when the polymer precipitates quickly, the same amount of dispersed phase will result in larger microparticles than when it solidifies slowly. In this case, the polymer matrix is not as dense as when it is allowed to shrink for a longer period of time (Fig. 4-B). However, the space present in the matrix is not as large as the pores created by water influx but small enough to function as a diffusional barrier to the protein. To control the release of BSA, for example, of which the effective molecular radius is $\sim 3.6 \mathrm{~nm}$, the dispersed phase is supposed to shrink to such an extent that the "pores" are also on a nanometer scale. If solidification occurs too fast,

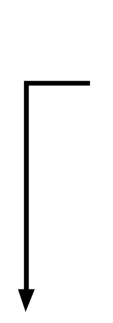

(A) Slow Solidification

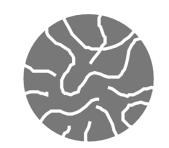

Porous microparticle

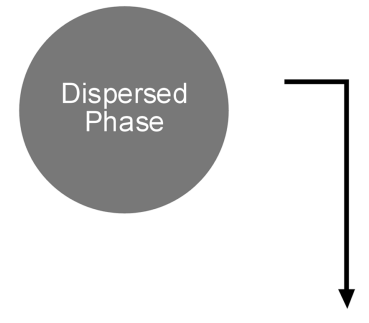

(B) Fast Solidification

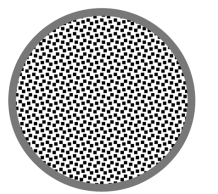

Low density microparticle
Fig. 4. Schematic description of the relationship between solidification rate and the particle morphology

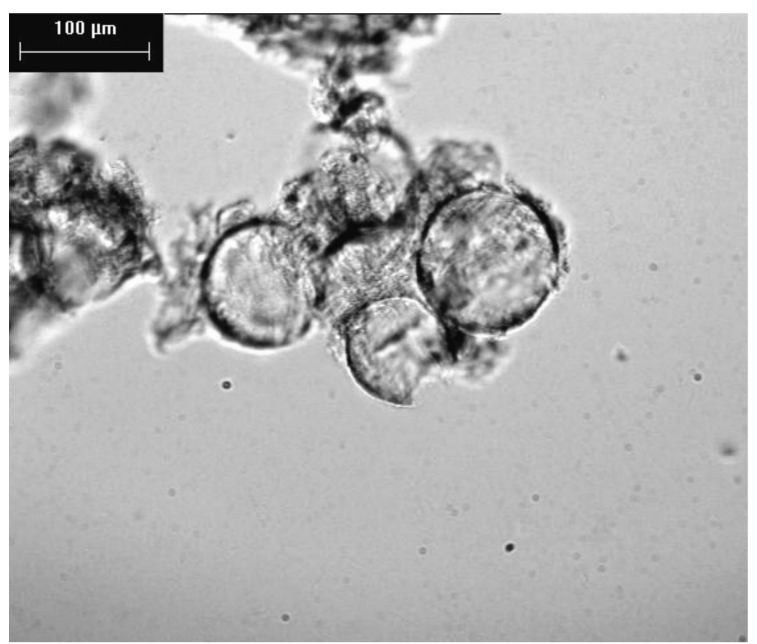

Fig. 5. Microparticles formed by the solvent exchange method. Acetic acid was used as a solvent for the polymer. Due to the instant precipitation of the polymer, the polymer membranes covering the aqueous cores became a collection of discontinuous polymer precipitates. See Reference (Yeo et al., 2003).

the precipitating polymer cannot make up for the space left after extraction of the solvent and ends up being a collection of continuous polymer islets. An exemplary case would be when the organic solvent for the polymer is highly water-soluble or water-miscible (Fig. 5).

In summary, the relationship between solidification rate and particle morphology is as follows:

(a) When solidification is slow, water influx from the continuous phase and/or the internal aqueous phase into the polymer phase create water-filled channels or pores. Microparticles shrink further during the time allowed for solidification and become dense and small. Generally, small porous microparticles have a large surface area and, hence, result in low encapsulation efficiency and high initial burst (Jiang et al., 2002).

(b) When solidification is moderately fast, water does not flow into the dispersed phase substantially, but the dispersed phase can shrink to make the matrix moderately dense. These microparticles are larger than those resulting from slow solidification. Their encapsulation efficiencies are high, and initial bursts are low. When fast solidification occurs due to the high polymer concentration, the polymer matrix will be dense because of the chain entanglement (Yang et al., 2001).

(c) When solidification is too fast, the dispersed phase will instantly solidify upon contact with the continuous phase without any volumetric change. Here, the solvent will be removed by quick solvent extraction into the continuous phase and leave a collection of polymer islets. In this case, the polymer precipitate may be unable to control the release rate and result in a large burst release.

A study of PLGA microparticles containing SCT provides 
an example relevant to the above discussion (Jeyanthi et al., 1997). PLGA microparticles were prepared by dispersing a mixture of PLGA-methylene chloride solution and SCTmethanol solution in an aqueous continuous phase. As the encapsulated SCT increased, the specific surface area of the microparticles increased, which indicated increase in porosity. According to the authors, the increased porosity was related to removal of methanol and subsequent replacement of the methanol with water. Increase of the peptide concentration increased the amount of methanol remaining in the microparticles. Increasing methanol contents facilitated water influx, which usually occurs when solidification of the dispersed phase is delayed. On the other hand, as a non-solvent for the polymer, methanol caused fast solidification of the dispersed phase. In this case, the microcapsules displayed characteristics of both slow solidification (high porosity) and fast solidification (large particle size).

\section{Formulation parameters affecting burst release}

When initial burst is mainly due to drug diffusion through the preformed water channels, the solidification rate of the dispersed phase is a critical factor that influences the initial release. Therefore, most formulation parameters discussed above are involved in control of the initial burst as well.

\section{Molecular weight of polymer}

Initial burst is affected by the molecular weight of the polymer. In general, low molecular weight polymers result in high burst release of encapsulated protein (Yang et al., 2001, O'Hagan et al., 1994, Cohen et al., 1991, Kissel et al., 1996). This is partly because the low molecular weight polymer is more soluble in the organic solvent and undergoes slow solidification to produce more porous microparticles. On the other hand, it can also be attributed to the smaller size of the microparticles, which provides more surface area for drug diffusion (Yang et al., 2001).

\section{Polymer concentration}

A low polymer concentration results in high internal porosity and, thus, high initial bursts. A dilute dispersed phase can introduce more water from the continuous phase and/or the internal water phase and create water pores or channels before it completely solidifies. Once the microparticles are dried, the water channels become hollow holes through which drugs can be burst-released (Yang et al., 2000). High porosity of the microparticles made of a low concentration polymer solution was visualized using confocal microscopy (Yang et al., 2001). Due to the high porosity, the microparticles resulted in a steep release profile.

The same trend was observed in PLGA (L/G ratio=75/
25) microparticles loaded with BSA (Sah et al., 1994). The extent of initial burst decreased with increasing polymer concentration. However, the release pattern consisted of an initial burst followed by little subsequent release, and it was common for all levels of PLGA concentrations. The release rate was controlled only by blending a fastdegrading low molecular weight d,I-PLA with the PLGA (75/25). This result indicates that the initial burst is a diffusion-based process, which is influenced by morphology of the microparticles while the subsequent release is a function of the polymer degradation.

\section{Hydrophilicity of polymer}

In general, hydrophilic polymers result in high initial burst and high release rates. For example, the extent of initial ovalbumin release was relatively high when PLGA with relatively high glycolide content $(50 / 50)$ was used (O'Hagan et al., 1994). The higher glycolide content made the polymer more hydrophilic, facilitated water uptake from the release medium, and resulted in a higher initial burst. Similarly, release of cyclosporine A from PLGA microparticles was affected by copolymer composition (Lee et al., 2002). Higher lactide content delayed the drug release because of the stronger hydrophobic interaction between the polymer and the drug. On the other hand, the slow drug release can also be attributed to the delayed hydration of the microparticles due to the hydrophobicity of the PLGA.

The end structure of PLGA polymer is another factor that affects the hydrophilicity of the polymer. In DNA microencapsulation, PLGA carrying free carboxylic end groups (Resomer ${ }^{\circledR} R G 502 H$ ) resulted in a higher encapsulation efficiency as well as a higher release rate, as compared to the end-capped polymer (Walter et al., 2001).

\section{Drug loading}

In many cases, the initial release increases with increasing protein loading (Sah et al., 1994; O'Hagan et al., 1994; Hora et al., 1990). There are two possible explanations for the effect of protein loading. First, the elution of surfaceassociated protein creates water-filled channels that allow subsequent elution of the proteins located inside the microparticles. By facilitating formation of these channels, high protein loadings lead to high initial bursts (Hora et al., 1990). Alternatively, a large protein concentration gradient between the microparticles and the release medium may promote the high initial bursts (Yang et al., 2001). However, different opinion exists: The concentration gradient does not influence the protein release until the onset of polymer degradation (Sah et al., 1994).

\section{Composition of continuous phase}

Variation of the PVA concentration in the continuous 
phase influences particle size, encapsulation efficiency, and/or initial burst (Hsu et al., 1999; Yang et al., 2001). The particle size decreases with increasing PVA concentration, due to the increased shear stress and viscosity. Despite the small size, these microparticles show decreased initial burst (Yang et al., 2001) or increased encapsulation efficiency (Hsu et al., 1999). It is supposed that high PVA concentration prevents migration of the internal aqueous phase toward the continuous phase by increasing the viscosity of the continuous phase (Yang et al., 2001).

Addition of salt or sugar into the continuous phase contributes to suppression of the initial burst (Jiang et al., 2002). According to Jiang et al., the initial burst decreased by $46 \%$ and $27 \%$ by addition of $\mathrm{NaCl}$ and sucrose, respectively. Here, the presence of salt or sugar increased the osmotic pressure across the polymer phase, which was in essence a semi-permeable membrane in the semisolid state. This increase in osmotic pressure prevented influx of the continuous phase into the dispersed phase and reduced the formation of water channels which could have led to a high initial burst. The flow of water from the internal aqueous phase $\left(w_{1}\right)$ into the continuous phase $\left(w_{2}\right)$ seemed to exert an insignificant effect on the initial release since the BSA, a model drug in this study, was unable to cross the "semi-permeable" polymer phase due to its size. On the other hand, in a different study on insulin-loaded PLA microparticles, the osmotic pressure gradient induced by the addition of $\mathrm{NaCl}$ led to diffusion of the $w_{1}$ phase into the $w_{2}$ phase, compromising the encapsulation efficiency (Uchida et al., 1997). Going back to our discussion on the initial burst, the difference between $\mathrm{NaCl}$ and sucrose in their capabilities of reducing the initial burst seems due to their different contributions to the continuous phase (Jiang et al., 2002). In addition to osmotic pressure, $\mathrm{NaCl}$ increases the polarity of the continuous phase. Since methylene chloride is less soluble in the polar continuous phase, the polymer precipitates more slowly and forms relatively dense microparticles. On the other hand, sucrose lacks this capability and, thus, is not as effective as $\mathrm{NaCl}$ in burst reduction.

\section{Drug distribution in microparticles}

Migration of drugs during drying and storage steps can result in a heterogeneous drug distribution in the polymer matrix (Huang and Brazel, 2001). During the air or vacuum drying process, in particular, water flows to the matrix surfaces before evaporation. Here, drugs also diffuse toward the surface by convection and result in heterogeneous drug distribution in the polymer matrix (Fig. 6).

It seems that convection-induced drug migration can be reduced by freeze-drying the microparticles. Burst release was lower when microparticles were freeze-dried than when air-dried (Igartua et al., 1997). In another example,

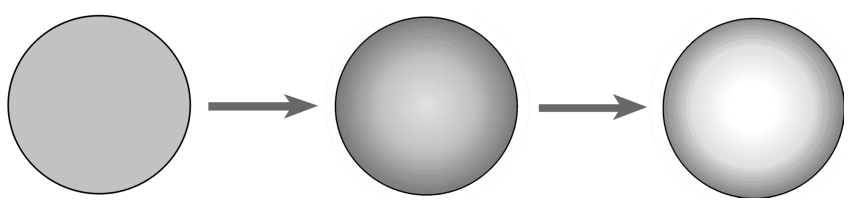

Fig. 6. Potential drug redistribution due to convection during the drying process (Modified from Reference (Huang and Brazel, 2001)). Shaded area indicates drug distribution.

the burst release was almost entirely eliminated by freeze-drying the microparticles (Wang et al., 1991). The authors attribute this result to the effectiveness of drying; however, suppressed migration of the protein during the drying process can also be a possibility. On the other hand, the freeze-drying process is not always effective in preventing the burst release. The volume expansion of encapsulated water during the freezing stage can cause additional cracks in the microparticles and induce a large initial burst.

As discussed in the previous section, when the drug is highly soluble in the continuous phase, the drug tends to migrate toward the exterior of the microparticles, also resulting in heterogeneous drug distribution. An example was shown in insulin microencapsulation using the solid in oil in water $(\mathrm{s} / \mathrm{o} / \mathrm{w})$ emulsion method (Yamaguchi et al., 2002). According to Yamaguchi et al., addition of a small fraction of glycerol into the primary ( $/ / 0$ ) suspension enhanced internalization of insulin into the PLGA microparticles, by forming a "mini-emulsion", and suppressed the initial burst from $40 \%$ to $10 \%$. The drug distribution was visualized by dying the microparticles with Coomassie Brilliant Blue, a protein-specific visible dye: The surface of the microparticles prepared in the presence of glycerol was virtually not stained, whereas the microparticles prepared without glycerol were homogeneously covered with the blue dye, which indicated presence of insulin on the surface of the microparticles. This result clearly shows that the affinity of drugs to the continuous phase can lead to highly heterogeneous drug distribution unless the imbalance of affinity is nullified by the presence of glycerol. The suppression of the initial burst is also attributed to the plasticizing effect of the glycerol. The presence of glycerol decreased glass transition temperature $\left(T_{g}\right)$ of PLGA microparticles from $42.5^{\circ} \mathrm{C}$ to $36.7^{\circ} \mathrm{C}$, making the polymer rubbery at physiological temperature $\left(37^{\circ} \mathrm{C}\right)$. SEM images show that the pores originally present on the surface of the microparticle disappeared after 4-h incubation in a phosphate buffer at $37^{\circ} \mathrm{C}$ : The pores collapsed during the incubation due to depression of $\mathrm{T}_{\mathrm{g}}$. By blocking the pores through which the encapsulated insulin can be burst released, the presence of glycerol contributed to suppression of the initial burst.

Similarly, human serum albumin was more efficiently 
Table IV. Possible strategies to reduce the initial burst (Huang and Brazel, 2001)

- In vitro extraction of surface-bound drugs for a short period of time prior to in vivo application.

- Additional coating to provide a blank outer layer.

- Non-uniform drug distribution so that concentrations may gradually increase from the surface to the core (effective in monolithic systems).

- Variation of polymer composition.

- Surface modification.

internalized within PLA-PEG copolymer (PELA) than in PLGA polymer (Li et al., 2001). The initial burst and the "surface-associated protein", represented by the protein releasable upon trypsin treatment, were significantly reduced by using PELA polymer. The contribution of PELA polymer is due to the relatively hydrophilic microenvironment created by the PEG segment, in which the encapsulated protein can easily reside.

The efficient internalization of the drug can also be achieved by generating a fine primary emulsion (Sah et al., 1995; Yan et al., 1994). The fine primary emulsion was obtained using a high energy homogenization method such as sonication (Yan et al., 1994) or high shear rate application (Sah et al., 1995). Homogeneous drug distribution was demonstrated by confocal microscopy (Yan et al., 1994) or SEM (Sah et al., 1995).

\section{Approaches to reduce initial burst}

Different approaches have been attempted to reduce the initial burst. A recent publication thoroughly reviews existing approaches (Huang and Brazel, 2001). Representative examples are listed in Table IV. Most examples focused on reducing the initial burst associated with the surface-bound drugs.

\section{CONCLUSIONS}

Initial burst is usually undesirable because the drug released in this period is not available for prolonged release, and, more importantly, it can result in toxic side effects. In order to prevent the initial burst and gain efficient control over the release rate, it is necessary to understand possible causes of the initial release and relevant formulation parameters. In general, initial burst depends on how efficiently the drug is captured within the microparticles. Encapsulation efficiency is another aspect of efficient drug capture; therefore, the formulation parameters that govern the initial burst and the encapsulation efficiency are mostly overlapped.

The present article provided a review of several formulation variables that affect encapsulation efficiency and initial burst, focusing on protein-loaded microparticles. Possible causes of the initial burst and existing approaches to decrease the burst releases were also discussed. Protein release out of biodegradable polymeric devices is dependent on diffusional escape of the protein prior to the onset of polymer degradation. Poor control over the diffusion during the initial stage results in large initial burst and premature depletion of the drug. In order to control the release profiles efficiently, it is important that the microparticles have appropriate morphological characteristics, such as pore distribution and average pore size, and that polymers having a desirable degradation profile are used.

\section{REFERENCES}

Alkermes, Medisorb Polymer Products (http://www.alkermes. com/polymer/products.html).

Bodmeier, R. and McGinity, J. W., Solvent selection in the preparation of PLA microspheres prepared by the solvent evaporation method. Int. J. Pharm., 43, 179-186 (1988).

Boury, F., Marchais, H., Proust, J. E., and Benoit, J. P., Bovine serum albumin release from poly(alpha-hydroxy acid) microspheres: effects of polymer molecular weight and surface properties. J. Controlled Release, 45, 75-86 (1997).

Cohen, S., Yoshioka, T., Lucarelli, M., Hwang, L. H., and Langer, R., Controlled delivery systems for proteins based on PLGA microspheres. Pharm. Res., 8, 713-720 (1991).

Crotts, G. and Park, T. G., Stability and release of bovine serum albumin encapsulated within PLGA microparticles. J. Controlled Release, 44, 123-134 (1997).

Frauke Pistel, K., Breitenbach, A., Zange-Volland, R., and Kissel, T., Brush-like branched biodegradable polyesters. Part III. Protein release from microspheres of poly(vinyl alcohol)-graft-poly(D,L-lactic-co-glycolic acid). J. Controlled Release, 73, 7-20 (2001).

Hora, M. S., Rana, R. K., Nunberg, J. H., Tice, T. R., Gilley, R. M., and Hudson, M. E., Release of human serum albumin from PLGA microspheres. Pharm. Res., 7, 1190-1194 (1990).

Hsu, Y.-Y., Hao, T., and Hedley, M. L., Comparison of process parameters for microencapsulation of plasmid DNA in poly(D,L-lactic-co-glycolic) acid microspheres. J. Drug Targeting, 7, 313-323 (1999).

Huang, X. and Brazel, C. S., On the importance and mechanisms of burst release in matrix-controlled drug delivery systems. J. Controlled Release, 73, 121-136 (2001).

Igartua, M., Hernandez, R. M., Esquisabel, A., Gascon, A. R., Calvo, M. B., and Pedraz, J. L., Influence of formulation variables on the in vitro release of albumin from biodegradable microparticulate systems. J. Microencapsulation, 14, 349-356 (1997).

Jeyanthi, R., Mehta, R. C., Thanoo, B. C., and DeLuca, P. P., Effect of processing parameters on the properties of peptidecontaining PLGA microspheres. J. Microencapsulation, 14, 163-174 (1997). 
Jeyanthi, R., Thanoo, B. C., Metha, R. C., and DeLuca, P. P., Effect of solvent removal technique on the matrix characteristics of polylactide/glycolide microspheres for peptide delivery. J. Controlled Release, 38, 235-244 (1996).

Jiang, G., Thanoo, B. C., and DeLuca, P. P., Effect of osmotic pressure in the solvent extraction phase on BSA release profile from PLGA microspheres. Pharm. Dev. Technol., 7, 391-399 (2002).

Johansen, P., Men, Y., Audran, R., Corradin, G., Merkle, H. P., and Gander, B., Improving stability and release kinetics of microencapsulated tetanus toxoid by co-encapsulation of additives. Pharm. Res., 15, 1103-1110 (1998).

Kim, H. K. and Park, T. G., Microencapsulation of human growth hormone within biodegradable polyester microspheres: protein aggregation stability and incomplete release mechanism. Biotechnol. Bioeng., 65, 659-667 (1999).

Kissel, T., Y., X. L., Volland, C., Gorich, S., and Koneberg, R., Parenteral protein delivery systems using biodegradable polyesters of $A B A$ block structure, containing hydrophobic poly(lactide-co-glycolide) A blocks and hydrophilic poly(ethylene oxide) B blocks. J. Controlled Release, 39, 315326 (1996).

Lee, W.-k., Park, J.-y., Yang, E. H., Suh, H., Kim, S. H., Chung, D. S., Choi, K., Yang, C. W., and Park, J.-S., Investigation of the factors influencing the release rates of cyclosporin $A$ loaded micro- and nanoparticles prepared by high-pressure homogenizer. J. Controlled Release, 84, 115-123 (2002).

Li, W.-I., Anderson, K. W., Mehta, R. C., and DeLuca, P. P., Prediction of solvent removal profile and effect on properties for peptide-loaded PLGA microspheres prepared by solvent extraction/evaporation method. J. Controlled Release, 37, 199-214 (1995).

Li, X., Deng, X., and Huang, Z., In Vitro Protein Release and Degradation of Poly-D,L-lactide-poly(ethylene glycol) Microspheres with Entrapped Human Serum Albumin. Pharm. Res., 18, 117-124 (2001).

Li, X., Deng, X., Yuan, M., Xiong, C., Huang, Z., Zhang, Y., and Jia, W., Investigation on process parameters involved in preparation of polylactide-poly(ethylene glycol) microspheres containing Leptospira Interrogans antigens. Int. J. Pharm., 178, 245-255 (1999).

Lu, W. and Park, T. G., Protein release from poly(lactic-coglycolic acid) microspheres: protein stability problems. PDA J. Pharm. Sci. Technol., 49, 13-19 (1995).

Maa, Y.-F. and Hsu, C. C., Effect of primary emulsions on microsphere size and protein-loading in the double emulsion process. J. Microencapsulation, 14, 225-241 (1997).

Mehta, R. C., Jeyanthi, R., Calis, S., Thanoo, B. C., Burton, K. W., and DeLuca, P. P., Biodegradable microspheres as depot system for parenteral delivery of peptide drugs. J. Controlled Release, 29, 375-384 (1994).

Mehta, R. C., Thanoo, B. C., and DeLuca, P. P., Peptide containing microspheres from low molecular weight and hydrophilic
poly(D,L-lactide-co-glycolide). J. Controlled Release, 41, 249257 (1996).

Nihant, N., Schugens, C., Grandfils, C., Jerome, R., and Teyssie, P., Polylactide microparticles prepared by double emulsion/ evaporation technique. I. Effect of primary emulsion stability. Pharm. Res., 11, 1479-1484 (1994).

Nihant, N., Schugens, C., Grandfils, C., Jerome, R., and Teyssie, P., Polylactide microparticles prepared by double emulsionevaporation. II. Effect of the poly (lactide-co-glycolide) composition on the stability of the primary and secondary emulsions. J. Colloid Interface Sci., 173, 55-65 (1995).

Ogawa, Y., Yamamoto, M., Okada, H., Yashiki, T., and Shimamoto, T., A new technique to efficiently entrap leuprolide acetate into microcapsules of polylactic acid or copoly(lactic/glycolic) acid. Chem. Pharm. Bull., 36, 1095-1103 (1988).

O'Hagan, D. T., Jeffery, H., and Davis, S. S., The preparation and characterization of PLGA microspheres: III. Microparticle/ polymer degradation rates and the in vitro release of a model protein. Int. J. Pharm., 103, $37-45$ (1994).

Park, T. G., Lee, H. Y., and Nam, Y. S., A new preparation method for protein loaded poly(D,L-lactic-co-glycolic acid) microspheres and protein release mechanism study. J. Controlled Release, 55, 181-191 (1998).

Rafati, H., Coombes, A. G. A., Adler, J., Holland, J., and Davis, S. S., Protein-loaded PLGA microparticles for oral administration: formulation, structural and release characteristics. $J$. Controlled Release, 43, 89-102 (1997).

Sah, H., Microencapsulation techniques using ethyl acetate as a dispersed solvent: effects of its extraction rate on the characteristics of PLGA microspheres. J. Controlled Release, 47, 233-245 (1997).

Sah, H., Toddywala, R., and Chien, Y. W., The influence of biodegradable microcapsule formulations on the controlled release of a protein. J. Controlled Release, 30, 201-211 (1994).

Sah, H. K., Toddywala, R., and Chien, Y. W., Biodegradable microcapsules prepared by a w/o/w technique: effects of shear force to make a primary w/o emulsion on their morphology and protein release. J. Microencapsulation, 12, 59-69 (1995).

Schlicher, E. J. A. M., Postma, N. S., Zuidema, J., Talsma, H., and Hennink, W. E., Preparation and characterization of poly(D,L-lactic-co-glycolic acid) microspheres containing desferrioxamine. Int. J. Pharm., 153, 235-245 (1997).

Schugens, C., Laruelle, N., Nihant, N., Grandfils, C., Jerome, R., and Teyssie, P., Effect of the emulsion stability on the morphology and porosity of semicrystalline poly L-lactide microparticles prepared by $\mathrm{w} / \mathrm{o} / \mathrm{w}$ double emulsion-evaporation. J. Controlled Release, 32, 161-176 (1994).

Schwendeman, S. P., Recent advances in the stabilization of proteins encapsulated in injectable PLGA delivery systems. Crit Rev Ther Drug Carrier Syst, 19, 73-98 (2002).

Sinisterra, R. D., Shastri, V. P., Najjar, R., and Langer, R., Encapsulation and release of rhodium(II) citrate and its 
association complex with hydroxypropyl- $\beta$-cyclodextrin from biodegradable polymer microspheres. J. Pharm. Sci., 88, 574-576 (1999).

Tracy, M. A., Development and scale-up of a microsphere protein delivery system. Biotechnol. Prog., 14, 108-115 (1998).

Uchida, T., Nagareya, N., Sakakibara, S., Konishi, Y., Nakai, A., Nishikata, M., Matsuyama, K., and Fukada, K., Preparation and characterization of polylactic acid microspheres containing bovine insulin by a w/o/w emulsion solvent evaporation method. Chem. Pharm. Bull., 45, 1539-1543 (1997).

van de Weert, M., Hennink, W. E., and Jiskoot, W., Protein instability in PLGA microparticles. Pharm. Res., 17, 11591167 (2000a).

van de Weert, M., van't Hof, R., van der Weerd, J., Heeren, R. M. A., Posthuma, G., Hennink, W. E., and Crommelin, D. J. A., Lysozyme distribution and conformation in a biodegradable polymer matrix as determined by FT-IR techniques. $J$. Controlled Release, 68, 31-40 (2000b).

Walter, E., Dreher, D., Kok, M., Thiele, L., Kiama, S. G., Gehr, P., and Merkle, H. P., Hydrophilic poly (D,L-lactide-co-glycolide) microspheres for the delivery of DNA to human-derived macrophages and dendritic cells. J. Controlled Release, 76, 149-168 (2001).

Wang, H. T., Schmitt, E., Flanagan, D. R., and Linhardt, R. J., Influence of formulation methods on the in vitro controlled release of protein from poly(ester) microspheres. J. Controlled Release, 17, 23-32 (1991).
Yamaguchi, Y., Takenaga, M., Kitagawa, A., Ogawa, Y., Mizushima, Y., and Igarashi, R., Insulin-loaded biodegradable PLGA microcapsules: initial burst release controlled by hydrophilic additives. J. Controlled Release, 81, 235-249 (2002).

Yan, C., Resau, J. H., Hewetson, J., Mest, M., Rill, W. L., and Kende, M., Characterization and morphological analysis of protein-loaded poly(lactide-co-glycolide) microparticles prepared by water-in-oil-in-water emulsion technique. J. Controlled Release, 32, 231-241 (1994).

Yang, Y.-Y., Chia, H.-H., and Chung, T.-S., Effect of preparation temperature on the characteristics and release profiles of PLGA microspheres containing protein fabricated by doubleemulsion solvent extraction/evaporation method. J. Controlled Release, 69, 81-96 (2000).

Yang, Y.-Y., Chung, T.-S., and Ping Ng, N., Morphology, drug distribution, and in vitro release profiles of biodegradable polymeric microspheres containing protein fabricated by double-emulsion solvent extraction/evaporation method. Biomaterials, 22, 231-241 (2001).

Yeo, Y., Basaran, O. A., and Park, K., A new process for making reservoir-type microcapsules using ink-jet technology and interfacial phase separation. J. Controlled Release, 93, 161173 (2003).

Zhu, G. and Schwendeman, S. P., Stabilization of proteins encapsulated in cylindrical PLGA implants: mechanism of stabilization by basic additives. Pharm. Res., 17, 351-357 (2000). 